McElligott, G. L. M. (1943) Brit. med. J., 1, 296.

Medical Research Council (1943) War Memorandum, No. 10.

Ministry of War Transport (1943) Notice to Ships' Masters, No. M.237.

Northey, E. H. (1943) Industr. Engng Chem., 35, 829.

Pappas, J. P. (1942) Milit. Surg., 90, 45.

Petro, J. (1943) Lancet, 1, 35.

Priest, W. H. D. (1943) J. R. Army med. Corps, 81, 139.

Roblee, M. A. (1943) Amer. J. Obstet. Gynec., 46, 400.

Speert, H. (1943) Amer. J. Obstet. Gynec., 45, 200.

La Towsky, L. W., Knight, F., Uhle, C. A. W., and Baker, R. B. (1942) J. Lab. clin. Med., 27, 1001.

Tréfouêl, J., and Tréfouêl, Mme. J., Nitti, F., and Bovet, D. (1935) C. R. Soc. Biol., 120, 756.

Wyss, O., Strandskov, Frede B., and Schmelkes, F. C. (1942) Science, 96, 236.

\title{
SPECIFIC REACTIONS OCCURRING IN THE TREATMENT OF LYMPHOGRANULOMA. VENEREUM WITH IMMUNE ANIMAL - SERUMS
}

\author{
By F. SAGHER, M.D. \\ Dermatological Department of the Rothschild-Hadassah University Hospital, Jerusalem
}

We have already reported elsewhere upon the results of the treatment of lymphogranuloma venereum with human convalescent serum. It has been pointed out that the use of this serum, in view of the results of experiments on thirty patients, could be regarded as a specific therapy for that condition. It was shown that the illness could be curtailed and that recurrences were not observed after the treatment had been carried out in the way suggested. Usually the serum was administered intramuscularly. In a later series of another twenty patients similar results were recorded when the serum was given intravenously but no acceleration of the healing process could be achieved by this method. Pursuing this line of treatment further, we tried to substitute for the human serum that of animals because, on the one hand, human convalescent serum is not always available and on the other it was desirable to have a method at hand which could also be used in the treatment of out-patients. Although the results of sulphonamide therapy applied to this disease are excellent and have been reported, specific therapy has not lost anything of its importance.

\section{Report of experiments}

A detailed account of our experiments, which met with a considerable degree of difficulty, is here published because it may supply an interesting contribution to the still rather unclarified problem of immunity in animals as well as in humans.

Human convalescent serum, whether given by the intramuscular or intravenous route, had not in any case produced ill effects apart from one patient who developed a gluteal abscess. To obtain convalescent serum from animals it is necessary to produce an infection in the animal and to obtain the serum during the period of healing or after disappearance of the symptoms. Monkeys or rabbits lend themselves to these experiments since only in animals of their size has the production of symptoms of lymphogranuloma venereum been effected. Neither guineapigs nor mice could be considered since the yield of antiserum is so small. As to other bigger animals it was not certain whether the disease could be transmitted to them, and we could not find any reference to this matter in the literature at our disposal. Since, for financial reasons, monkeys were out of the question, we decided to use rabbits and sheep although positive transmission to sheep has not previously been described.

The experiments were carried out as follows. Pus was injected into sheep and rabbits. It was obtained from a patient suffering from lymphogranuloma venereum and was taken on the same day on which the first injection was to be given to the animals. Since the virus is known to maintain its virulence for a 
period not longer than eight days the animals had therefore on some occasions received injections of material which was not any longer virulent.

The following injections were given. Sheep were inoculated with an injection of 1 cubic centimetre of pus diluted in saline in the proportion of $1: 7 ;$ after ten days this was followed by 1 cubic centimetre of pus diluted in the proportion of $1: 4$; after a further period of ten days the same amount diluted in the proportion of $1: 2$ was given. These injections were given intravenously.

Three rabbits received the following quantities. (a) 0.5 cubic centimetre of pus intravenously in dilution $1: 7 ;(b)$ three injections of 0.5 cubic centimetre each of pus in dilution $1: 7$ at intervals of 5 days ; (c) six injections of pus in dilution $1: 7$ at similar intervals.

During the subsequent nine months symptoms of lymphogranuloma venereum were not developed by the animals. The third rabbit, which died shortly after the sixth injection, presented pneumonia but did not show any swelling of lymphatic glands or any other symptom suggestive of lymphogranuloma venereum. One month after inoculation the animals were bled and from the bloods we prepared about 100 cubic centimetre of rabbit and sheep serum respectively. The serum was found to be sterile when tested by inoculation into bouillon as well as on blood agar.

In order to ascertain the response to normal rabbit serum two patients suffering from lymphogranuloma venereum were given 50 and 40 cubic centimetres respectively of rabbit serum intravenously in doses of 10 and 20 cubic centimetres distributed over a period of eight days. These injections provoked no kind of reaction by the patients. Two other patients were then given injections of serum of the animals which had received preparatory treatment. For the sake of accuracy the histories of these patients are given below.

Case 1.-The patient was 26 years of age. In 1928 he contracted gonorrhoea and in 1931 syphilis. He had received four combined courses of bismuth salvarsan, the last injection of which was given in 1932 . The cerebrospinal fluid and the blood had been examined in another hospital with negative results. A fortnight before we saw him the patient had noticed swellings, which were increasing in size in both groins. Some days later there had formed on his penis a small ulcer which had, however, disappeared without treatment.

On admission to hospital on 15th January he presented a swelling in the left inguinal region, the size of a goose's egg, the overlying skin being markedly taut and inflamed; there was also some fluctuation. The swelling could be followed laterally along Poupart's ligament and, towards the midline, to Rosenmueller's gland. In the right inguinal region there were clusters of swollen glands the size of a fist, occupying the same area as on the left. Here the overlying skin was normal in appearance but was adherent to the swollen glands. In the iliac region the tumour had penetrated deeply. At the preputium we found an ulcer as big as a pea, with jagged edges and covered with a whitish-yellow discharge, but there was no infiltration. In the serum obtained by scarification of the sore, Spirochaeta pallida could not be identified, even upon repeated examination. The temperature was elevated, rising to $102 \cdot 5^{\circ} \mathrm{F}$. The Wassermann reaction was negative (Kaup's modification was slightly positive); the Meinicke flocculation test was negative; Mueller's agglutination test was positive ; the Frei test (intracutaneous) was repeatedly positive. The haemoglobin as estimated by the Sahli method was 80 per cent, colour index $1 \cdot 04$, erythrocytes $3,840,000$, leucocytes 13,400 , polymorphonuclears 71 per cent, lymphocytes 27 per cent, eosinophils 1 per cent, band forms 1 per cent, monocytes nil.

On 19th January puncture of the glands and withdrawal of 20 cubic centimetres of pus was effected. On 21 st January 20 cubic centimetres of the serum of one of the inoculated rabbits was given intravenously. Half an hour after the injection the patient complained of nausea, chills and perspiration, and there was pain in all the joints; the temperature rose to $104 \cdot 2^{\circ} \mathrm{F}$. After two hours, all these symptoms had disappeared. On 22 nd January the patient received 2 cubic centimetres of the same serum intravenously and there was no reaction. One hour later he was given 20 cubic centimetres of the same serum whereupon he developed symptoms similar to those of the previous day.

Since such unfavourable reactions had occurred when the patient was treated with immunized rabbit serum it was decided to ascertain the effect of normal rabbit serum. On 25th January therefore 5 cubic centimetres, and on 26th January 20 cubic centimetres of normal rabbit serum were administered intravenously ; no unfavourable reactions occurred.

It was now resolved to try the effect of using immuniźed sheep serum and human convalescent serum. On 27 th January 10 cubic centimetres of the serum of inoculated sheep, and on 28th January 10 cubic centimetres of human convalescent serum were given intravenously but no untoward reaction followed. As a result of the experience gained by using normal rabbit serum, immunized sheep serum and human convalescent serum, none of 
which produced any toxic effects, it was decided to return to the use of immunized rabbit serum. Hence on 31st January 10 cubic centimetres of the serum of an immunized rabbit were given intravenously, after a preparatory injection of 1 cubic centimetre to prevent the occurrence of an anaphylactic shock, whereupon the same serious symptoms appeared as those observed on 21st and 22nd January.

In view of these results it was finally decided to discontinue the use of immunized rabbit serum ; from 2nd February to 26th February the patient was given a total of 66 cubic centimetres of human convalescent serum intravenously, in doses of 8 to 17 cubic centimetres at a time, and untoward reactions were not observed. On 27th February, the patient was discharged from the hospital but advised to call for ambulatory treatment. During this period of after-treatment he did not show any untoward reaction to further administrations of human convalescent serum.

TABLE 1-CHART OF CASE 1

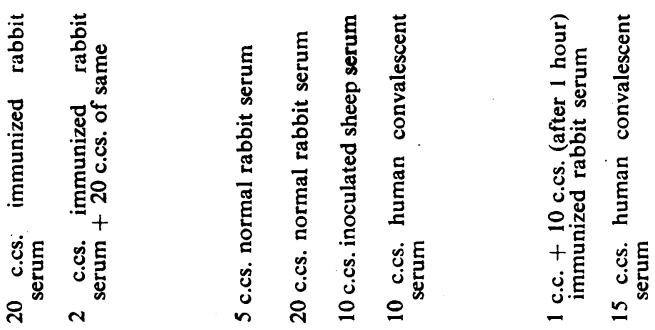

\begin{tabular}{|c|c|c|c|c|c|c|c|c|c|c|c|c|c|c|c|c|c|c|c|c|}
\hline Month & \multicolumn{16}{|c|}{ January } & \multicolumn{4}{|c|}{ Febucary } \\
\hline Day & 16 & 17 & 18 & 19 & 20 & 21 & 22 & 23 & 24 & 25 & 26 & 27 & 28 & 29 & 30 & 31 & 1 & 2 & 3 & 4 \\
\hline 106 & & & & & & & & & & & & $\vdots$ & & & & & & & & \\
\hline 105 & & & & & & & & & $\therefore$ & & & : & $:$ & & & & & & $\vdots$ & $\vdots$ \\
\hline 104 & & & & & & & & & & $\vdots \vdots$ & & $\vdots$ & & $!$ & $\vdots$ & $\div$ & & & $\vdots$ & \\
\hline 103 & & & & & & & & & & $\vdots$ & & 0 & & & & 18 & & & $\vdots$ & \\
\hline 102 & & $\vdots$ & & & & & $\vdots$ & & & $\vdots$ & & & & & & : & & & & \\
\hline 101 & & & & & & $t$ & $\vdots$ & & $\vdots$ & i- & & & & & & 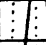 & & : & & $\vdots$ \\
\hline 100 & & : & & : & & it & $\therefore$ & & & 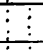 & $\vdots$ & $\frac{1}{\vdots}$ & & & & $\vdots$ & 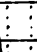 & & $\vdots$ & \\
\hline 99 & & & & & & & $:$ & & & & & & & & & & & & & \\
\hline & & & & 5 & & 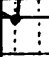 & & & & & & & & & & $\vdots$ & & ! & 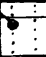 & \\
\hline & 2 & 3 & 4 & 5 & 6 & 7 & 8 & 9 & 10 & 11 & 12 & 13 & 14 & 15 & 16 & 17 & 18 & 19 & 20 & 21 \\
\hline
\end{tabular}

Case 2.-The patient, aged 32 years, had had syphilis in 1930. She received five courses of bismuth combined with salvarsan, the last injection having been given in May, 1933. Two years later and five days prior to her admission to hospital she observed a swelling of the lymphatic glands in the right inguinal region and had experienced a sensation of burning in the vagina. Her state on admission was as follows. In the vagina, just above the posterior commissure, there was a sharply outlined ulcer 1 centimetre in diameter, covered with a fatty discharge, and with a slight induration of the edges. Examination for $S$. pallida on several occasions was negative. In the right inguinal region there was a swelling the size of a hen's egg ; this was not tender however. The overlying skin was normal. The Wassermann, Meinicke and Mueller's tests were negative on three occasions. The patient was discharged from hospital, but when readmitted one month later she presented, in addition to the signs and symptoms previously noted, a swelling of the right labium major. No Ducrey's bacilli or $S$. pallida were yielded by the ulcer or by the lymphatic glands. Dmelcos injected intracutaneously did not give any reaction. Tubercle bacilli were not found. The Frei test (intracutaneous) was markedly positive (several repetitions). X-ray findings of the lungs showed calcified patches in the lower and central portions of both lungs, one small fibrous focus in the right apex as well as calcified broncho-pneumonic lymphatic glands on both sides. Basal adhesions were present on the right side ; the heart was normal.

The patient was given one combined course of bismuth salvarsan which did not provoke any ill effect. On 1st March she received 10 cubic centimetres of normal sheep's serum after a preparatory injection of 1 cubic centimetre. No unfavourable reaction was noted. On 2nd March she was given 10 cubic centimetres of immunized sheep serum intravenously without any ill effects. On 5th March she received 10 cubic centimetres of immunized rabbit serum whereupon a violent reaction developed ; the pulse vanished and cyanosis and fainting occurred immediately after injection. On 8th March a serum exanthema appeared. 
Each day for three days the patient was given 10 cubic centimetres of calcium intravenously ; by 10th March the skin symptoms had subsided. On 13th March puncturing of the fluctuating lymphatic gland led to the evacuation of 4 cubic centimetres of pus. The patient then received 10 cubic centimetres of human convalescent serum which was well tolerated. On 19th March she was given 25 cubic cèntimetres of human convalescent serum and immediately developed the same serious symptoms as on 5th March. At her own wish, the patient was allowed to return home but remained under supervision.

TABLE 2-CHART OF CASE 2
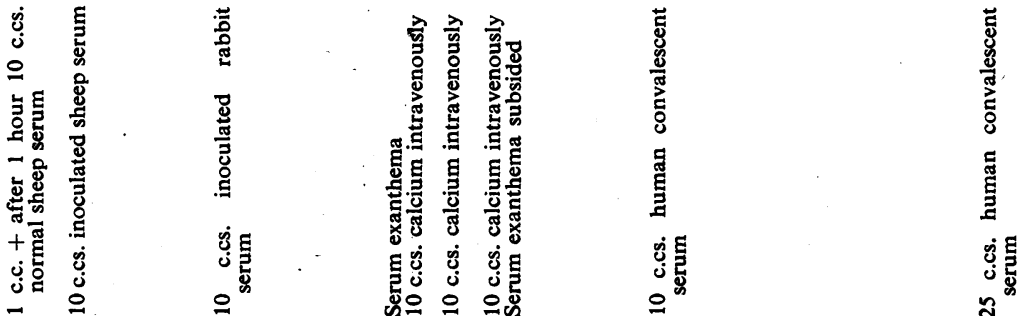

\begin{tabular}{|c|c|c|c|c|c|c|c|c|c|c|c|c|c|c|c|c|c|c|c|c|}
\hline Month & \multicolumn{20}{|c|}{ march } \\
\hline Day & 1 & 2 & 3 & 4 & 5 & 6 & 7 & 8 & 9 & 10 & 11 & 12 & 13 & $1 / 4$ & 15 & 16 & 17 & 18 & 19 & 20 \\
\hline 106 & & & $\vdots$ & & & & & & & & $\vdots \vdots \vdots$ & & $\vdots$ & & $\vdots \vdots$ & $\vdots \vdots \vdots \vdots$ & $\vdots$ & & & $\vdots$ \\
\hline 105 & & : & $\vdots$ & & & : & $\vdots$ & $\vdots$ & & & $\vdots \vdots$ & $\vdots \vdots$ & $\vdots \vdots \vdots$ & $\vdots$ & $\vdots$ & $\vdots \vdots \vdots$ & $\vdots$ & $\vdots \vdots \vdots$ & & $\vdots$ \\
\hline 104 & & & & & & & & $\vdots$ & & & $\vdots \vdots$ & & & & & $\begin{array}{l}\vdots \vdots \\
\end{array}$ & & & & $\vdots$ \\
\hline 103 & & & & & & & : & & & & $\vdots$ & & & $\vdots$ & & & $\vdots \vdots$ & & $\vdots$ & $\vdots$ \\
\hline 102 & & & & & & & & & $\vdots$ & & $\vdots$ & & & & $\vdots \vdots$ & & $\vdots \vdots$ & & $\dot{9}$ & $\vdots$ \\
\hline 101 & & & : & & & $\vdots$ & $\vdots$ & $\vdots$ & $\vdots \vdots$ & & $\vdots \vdots$ & & $\vdots$ & & $\vdots$ & & $\vdots$ & & : & $\vdots$ \\
\hline 100 & & & $\vdots$ & : & & & $\because$ & & $\vdots$ & $\vdots$ & $\vdots$ & & & & & $\vdots \vdots$ & $\vdots \vdots$ & & & ! \\
\hline 99 & & & 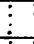 & & & $\vdots$ & $\vdots$ & & i & & $\bar{\vdots}$ & & & & & & & & & $\vdots$ \\
\hline 98 & & & & & & & & & & & & & & & & & & & & \\
\hline
\end{tabular}

The most striking features in both these cases were the serious complications produced by the injections of the serum of rabbits which had been inoculated with pus from lymphogranuloma venereum, although there was a slight difference in the character of the reactions in the two patients. In the first case the symptoms developed within from six minutes to half an hour after injection, beginning with a chill, followed by profuse perspiration and rise of temperature to $104 \cdot 2^{\circ} \mathrm{F}$. There was moreover generalized pain, but after two hours the patient was well again. In the case of the second patient the attack developed immediately. after injection and the condition was serious; the pulse was small, the face livid and pinched, the eyes turned upward and the woman lost consciousness. Later there was profuse sweating. The initial shock was so severe that we had to resort to cardiac stimulants, and after an hour she began to recover. The temperature did not rise so high as in the first case and declined during the course of the day.

\section{Commentary}

From the foregoing it appears that (1) injections of 10 to 20 cubic centimetres of serum from rabbits inoculated with pus from patients suffering from lymphogranuloma venereum provoked grave and nearly fatal reactions in patients suffering from this disease ; (2) that serum derived from immunized sheep provoked no reaction; (3) that a similar amount of serum from normal rabbits did not provoke any ill effects whatsoever. Our second patient could not be given normal rabbit serum, since she refused to consent to this procedure.

Once again it should be pointed out that all the serums we used were perfectly sterile and proved to be sterile even after a period of nine months by tests with bouillon cultures and on blood plates. Hence the ill effects occurring when 
immunized rabbit serum was given to patients suffering from lymphogranuloma venereum were not due to the fact that the serums were contaminated. In order to complete the chain of evidence the immunized rabbit serum should be given to healthy patients but this was not considered justifiable. It was however shown that normal rabbit serum proved harmless to some of our patients, when the doses had been distributed over ten injections, so that this source of error was also eliminated.

It would, however, be of the greatest interest to discover the factor responsible for the violent reactions reported above. Similar manifestations have been noted (1) in the vasomotor syndrome which occasionally occurs after intravenous injection of salvarsan, (2) in connexion with anaphylactic shock, (3) overdosage of vaccines or serums such as antidiphtheria serum and (4) as the result of lack of sterility of serums given by the intravenous route. The greatest care was taken in our cases that the serums should be perfectly sterile and as stated even after nine months their sterility was found to be maintained.

A vasomotor syndrome may be taken into consideration in view of the particular character of the symptoms, especially their immediate appearance in the second case as exemplified by the vanishing of the pulse and by the cyanosis. These angioneurotic shock symptoms have often been reported as occurring after injections of salvarsan and also as the result of the intravenous injection of other substances. There is always the possibility that the response of the patient to intravenous injections of serum in general might be different from that occurring upon injection of other substances. The first patient, however, did not show any particular response to injections of normal rabbit serum or to the injection of small quantities (10 cubic centimetres) of the serum of one of the treated animals. The symptoms only occurred upon injection of amounts exceeding 10 cubic centimetres. These reactions were not observed on any occasion upon injection of sheep serum. In the second case shock was reported after administration of the serum of treated rabbits although the serum of normal or treated sheep as well as neosalvarsan did not provoke any kind of untoward symptoms. To 10 cubic centimetres of human convalescent serum there was not any response but 25 cubic centimetres provoked a violent shock reaction similar to that which had occurred after the injection of the serum of treated rabbits. The latter fact seems to be of special significance; for previous to this investigation, in hundreds of cases, neither after intramuscular nor after intravenous injection of human convalescent serum had such a reaction been recorded. Another interesting point was that in this special case the convalescent serum had been obtained from patients who had been suffering from a particularly severe type of lymphogranuloma venereum and who had already recovered half a year ago. The serum of such patients was not generally available since there were not many who could be expected to volunteer for this action six months after they had been discharged from hospital.

Anaphylactic shock as the consequence of the administration of heterogeneous serum is, as a rule, not observed until the eighth day after the first injection. The majority of our injections were given before the eighth day and usually after a desensitizing injection of 1 to 2 cubic centimetres. If the condition presented by our patients was to be considered an anaphylactic shock reaction, a similar response to the administration of normal rabbit serum should be expected ; this, however, was not the case in any of the patients described.

The only possible explanation for this peculiar phenomenon, therefore, would be that the effects recorded by us were a direct result of the use of serum of the animals previously inoculated with pus from lymphogranuloma patients. It might be supposed that in the rabbit a slumbering infection had been provoked into activity and had rendered the serum capable of producing the serious symptoms in the patient to whom it was subsequently administered. If it were possible to identify in the animal specific antigens by means of the complement fixation test this would be evidence in favour of our assumption. In this way a latent infection in the animal might be proved, as it has already been proved for the human subject 
by Shaffer and his co-workers. The therapeutic value of the serum unfortunately could not be explored at the time.

There is also the possibility that we might have been faced with a re-transmission of the living serum which, in the meantime, would have multiplied in the animal's blood. A re-transmission to monkeys, which, indeed, would be the only definite proof of this theory, was impossible for technical reasons. Further experiments on the human subject had also to be abandoned in view of the seriousness of the symptoms provoked.

The objection might also be made that the rabbit, by treatment with human pus, may have been sensitized against human protein. This sensitized serum may subsequently have produced passive anaphylaxis in the human organism and the shock would therefore have to be considered a perfectly non-specific reaction. This theory, however, fails to disclose why any such manifestations were not observed in connexion with sheep serum. And further, the question remains unanswered as to why it is a dose of 25 cubic centimetres of human convalescent serum that should be the particular one to provoke the reaction. Therefore we think it likelier that we are dealing with a specific lymphogranuloma reaction due to overdosage of the serum of inoculated rabbits as well as human convalescent serum.

\section{Summary}

For experimental reasons, in order to ascertain whether there is a possibility of treating lymphogranuloma venereum with animal convalescent serum, three rabbits were injected intravenously with pus from lymphogranuloma patients. The animals did not develop any clinical symptoms. Upon treatment with serum derived from these animals two patients with lymphogranuloma venereum showed violent shock reactions, whereas normal rabbit serum had proved quite innocuous to these patients. Sheep serum obtained in a similar manner provoked no ill effects.

[These experiments were carried out at the Dermatological University Hospital in Prague with the assistance of the Department of Health of the Czechoslovak Government before the war.]

\section{REFERENCES}

Kalz, F. (1932) Derm. Wschr., 95, 1839.

- and Sagher, F. (1933) Derm. Wschr., 97, 1754.

Shaffer, M. F., Rake, G., Grace, A. W., McKee, Clara M., and Jones, Helen P. (1941) Amer. J. Syph., 25, 699.

\section{Syphilitic paroxysmal haemoglobinuria}

The Royal Australian Air Force Hospital in Sydney reports a case of paroxysmal haemoglobinuria from cold. An air observer, aged twenty-two years, had not any trace of syphilis and had been in good health. During his training in Canada he became very cold one day in his airplane and on landing passed red urine. Four days later the incident was repeated. When questioned he stated that between the ages of seven and twelve years he had passed red urine six or seven times during each winter when he had been extremely cold. An attack was produced by standing in a bucket of iced water for fifteen minutes. The Wassermann reaction and the Donath-Landsteiner test yielded positive results. The fundamental cause of this disease is syphilis; antisyphilitic treatment should be given for it sometimes brings relief.-Medical Journal of Australia, 18th December, 1943.

\section{Syphilis treated with phenarsine hydrochloride}

Boardman and Kaldeck report that a year and a half ago the treatment of syphilis with phenarsine hydrochloride was begun at Boston City Hospital. Ten to twenty injections, with alternating courses of fifteen injections of bismuth were given. Doses for men were from 0.030 to 0.067 gramme ; for women they were from 0.030 to 0.045 gramme. Severe gastro-intestinal reactions caused five patients to abandon treatment. Seven patients experienced such severé reactions that they had to give up treatment. Mild reactions occurred in many cases ; phenarsine was then given in smaller doses, with 10 cubic centimetres of either 50 per cent glucose or 10 per cent sodium thiosulphate. One patient in whom jaundice and hypocromic anaemia had developed completely recovered. There were not any deaths. The one case of primary syphilis which had not become negative had had only twelve injections of phenarsine and no bismuth during eight weeks. Lesions in primary and secondary syphilis healed in an average period of twenty-two days or $3 \cdot 14$ weeks. The percentage of serological reversals from positive to negative proved to be as large as that obtained with marpharsen and other arsenicals. - New England Journal of Medicine, 6th January, 1944. 